\title{
NOTE ON BLACK-BODY BRIGHTNESS EQUATIONS BASED ON EQUIVALENT WAVE-LENGTHS.
}

By Herbert E. Ives and E. F. Kingsbury.

IN a recent paper Foote and Fairchild ${ }^{1}$ develop equations for the luminous flux emitted by the black body by considering the flux a function of the equivalent wave-length. This mode of attack presupposes the existence of exact data derived by the more straightforward and logical method of integrating the product of emission by luminosity at each wave-length. The formulæ obtained by this indirect method are, however, considerably shorter than the formulæ necessary for the exact in tegration, ${ }^{2}$ and nearly as accurate.

The object of this note is to give the appropriate constants to represent by this new form of equation the results of using the spectral luminosity curve obtained by the present writers. ${ }^{2,3}$

Following the line of thought of Foote and Fairchild we note that the Wien equation for monochromatic intensity gives the following:

$$
\frac{d \log J}{d T}=\frac{c_{2}}{T^{2}} \frac{\mathbf{I}}{\lambda},
$$

where $J$ is monochromatic intensity, $T$ is absolute temperature, $c_{2}$ is the black body constant, and $\lambda$ is the wave-length observed. Now the full process of integration mentioned above shows that for $J$ we may substitute $L$, the luminous flux, provided we confine ourselves to a narrow range of temperature. The wave-length $\lambda$ then becomes the equivalent wave-length $\lambda_{e}$ for that temperature. This wave-length is not a constant but varies slowly with the temperature, and it is found that this variation may be quite closely represented by the equation.

$$
\lambda_{e}=a+\frac{b}{T}+\frac{c}{T^{2}}
$$

\footnotetext{
${ }^{1}$ Foote and Fairchild, "Luminosity of a Black Body and Temperature," Scientific Papers, Bureau of Standards, No. 270, March 21, r9I6.

${ }^{2}$ Kingsbury, "A Spectral Luminosity Curve and its Use," Physical Review, February, I9I6, p. I6r.

${ }^{3}$ Ives and Kingsbury, "Physical Photometry with a Thermopile Artificial Eye," PhysicaL Review, November, I9I5, p. 3I9.
} 
If this is substituted back in (I) we obtain as the luminous flux equation

$$
\frac{d \log L}{d T}=\frac{c_{2}}{a T^{2}+b T+c} .
$$

If $b$ and $c$ are taken as zero this is the Wien equation, which has been used in connection with the total light from the black body under the name of the Rasch equation. ${ }^{1}$ If $c$ alone is equal to zero, integration gives the Goldhammer or Nutting ${ }^{5}$ black body equation. When all three constants have real values there are three solutions, as follows:

$$
\begin{array}{ll}
L=A\left[\frac{T+\frac{b-\sqrt{b^{2}-4 a c}}{2 a}}{T+\frac{b+\sqrt{b^{2}-4 a c}}{2 a}}\right]^{\frac{c_{2}}{\sqrt{b^{2}-4 a c}}} & \text { if } b^{2}-4 a c>0, \\
L=A^{\prime} e^{\frac{2 c_{2}}{\sqrt{4 a c-b^{2}}} \tan -1}\left(\frac{2 a T^{\prime}+b}{\sqrt{4 a c-b 2}}\right) & \text { if } b^{2}-4 a c<0, \\
L=A^{\prime \prime} e^{-\frac{2 c_{2}}{2 a T^{2}+b}} & \text { if } b^{2}-4 a c=0 .
\end{array}
$$

Foote and Fairchild mention only the first of these, probably because the equivalent wave-length equation, which they derive by averaging several experimental spectral luminosity curves, happens to have the arrangement of signs and relative values of constants for which it is the solution.

On undertaking to put into this form the data calculated and tabulated by Kingsbury, ${ }^{2}$ embodying the experimental work of the writers, it was found that the equivalent wave-length equation was

$$
\lambda_{e}=.545+\frac{77}{T}-\frac{6000}{T^{2}}
$$

so that $b^{2}-4 a c$ is also positive for these data.

Using (7), equation (4) becomes

$$
L=23,934\left(\frac{T-55.853}{T+197.138}\right)^{104.221}
$$

which gives the light watts per steradian per unit solid angle with very great accuracy (within $\frac{1}{2}$ per cent.). From this the candlepower per square centimeter may be derived by dividing by the mechanical equivalent of light.

1 Rasch, Annalen d. Phys. (4), I4, p. I93, I904.

2 Nutting, "Luminosity and Temperature," Bull. Bur. Standards, No. 6, No. 3, p. 342, I909. 
Of the three expressions (4), (5) and (6), the third is by far the simplest, being in fact simpler to work with than the Nutting equation, which has the same number of parameters. The condition of its use $\left(b^{2}=4 a c\right)$ is not fulfilled by (7), but its simplicity has suggested that it be looked upon merely as another variation of the Wien or Rasch equation such as might have been suggested by knowledge of the manner in which that equation failed. We find that a slight variation of the constants needed for (7) gives in the following equation

$$
L=24,000 e^{-\frac{1}{.00008795 T^{T}+.00250}},
$$

a working formula correct to about $\frac{1}{2}$ per cent. over the range from I200$3000^{\circ}$.

The United Gas Improvement Company,

Physical Laboratory,

May I9, Igr6. 